\title{
Long non-coding RNA ZEBI-ASI promotes cell invasion and epithelial to mesenchymal transition through inducing ZEBI expression in cervical cancer
}

This article was published in the following Dove Press journal: OncoTargets and Therapy

\author{
Rongjie Chengl,* \\ $\mathrm{Nan} \mathrm{Li}^{2}, *$ \\ Shuyan Yang' \\ Lei Liu' \\ Shiyu Han'
}

'Department of Obstetrics and Gynaecology, The Fourth Affiliated Hospital of Harbin Medical University, Harbin City, Heilongjiang Province, People's Republic of China; ${ }^{2}$ Department of Pathology, The Fourth Affiliated Hospital of Harbin Medical University, Harbin City, Heilongjiang Province, People's Republic of China

*These authors contributed equally to this work
Correspondence: Shiyu Han Department of Obstetrics and Gynaecology, The Fourth Affiliated Hospital of Harbin Medical University, No 37 Yiyuan Road, Nangang District, Harbin I5000I, Heilongjiang Province, People's Republic of China Email uvyajb4@I63.com

\begin{abstract}
Background: Long non-coding RNAs (lncRNAs) play important roles in cancer initiation and development. The purpose of the present study was to determine the functions and mechanisms of lncRNA ZEB1-AS1 in human cervical cancer (CC).

Methods: A total of 106 pairs of $\mathrm{CC}$ tissues and adjacent normal epithelial tissues were collected from CC patients who underwent resection. Three human CC cell lines (HeLa, C33A and SiHa) and a normal cervical cell line Crl-2614 and were transfected with human ZEB1-AS1 cDNA, or empty vector as the control. Then, cells were transfected with ZEB1-AS1-specific small interfering RNA (si-ZEB1-AS1), ZEB1-specific siRNA (si-ZEB1) or negative siRNA control (si-NC). The transfection efficiency was confirmed by RT-qPCR analysis. qPCR was applied to determine the qualification of RNA. Cell proliferation was investigated by MTT assay. The apoptosis rate of cells was detected by flow cytometer. Cell invasion was detected by transwell assay. Western blot was applied to determine the expression of proteins. CC xenografts in 12 male BALB/c athymic nude mice were established. And the tumor volumes were measured by vernier caliper.

Results: We found that ZEB1-AS1 expression was remarkably increased in human CC tissue samples and cell lines, and its expression levels were closely associated with poor prognosis of CC patients. Moreover, we found that knockdown of ZEB1-AS1 inhibited the proliferation, migration, invasion and epithelial-mesenchymal transition (EMT) of CC cells in vitro and suppressed CC xenograft tumor growth in vivo. Mechanistically, we found that knockdown of ZEB1-AS1 significantly inhibited ZEB1 expression, and knockdown of ZEB1 could rescue the effects of ZEB1-AS1 overexpression in CC cells.
\end{abstract}

Conclusion: In conclusion, our findings indicated that ZEB1-AS1 serves an oncogenic role in $\mathrm{CC}$, which might become a potential prognostic indicator and therapeutic target in $\mathrm{CC}$.

Keywords: cervical cancer, long non-coding RNA, ZEB1-AS1, epithelial-mesenchymal transition, ZEB1

\section{Introduction}

Cervical cancer (CC) is one of the most common malignant cancers among women in both developed and developing countries. ${ }^{1}$ Primary treatments for CC include surgery, chemotherapy and radiotherapy. Despite encouraging advances in diagnostic and therapeutic strategies, the 5-year survival rate of patients with advanced/recurrent CC remains at only $10 \%-20 \% .{ }^{2}$ Therefore, exploring the molecular mechanisms underlying $\mathrm{CC}$ initiation and development has great significance for the treatment of this malignant disease. 
Recently, accumulating studies indicated that long noncoding RNAs (lncRNAs), a group of large transcripts ( $>200$ nucleotides in length) with limited or no protein-coding potential, serve critical roles in a wide variety of human diseases, particularly in cancers. ${ }^{3,4}$ Zinc finger E-box binding homeobox 1 antisense 1 (ZEB1-AS1), located in physical contiguity with ZEB1, is a cancer-related lncRNA and serves as an oncogenic regulator in diverse cancers, including glioma, ${ }^{5}$ colorectal cancer ${ }^{6}$ and gastric cancer. ${ }^{7}$ However, there are no reports regarding the role of ZEB1-AS1 in CC.

The present study might be the first one to elucidate the biological role and underlying mechanisms of ZEB1-AS1 in CC. We also investigated whether the effects of ZEB1-AS1 in $\mathrm{CC}$ were achieved by its regulation of ZEB1.

\section{Materials and methods}

\section{Patients and tissue samples}

A total of 106 pairs of $\mathrm{CC}$ tissues and adjacent normal epithelial tissues were collected from $\mathrm{CC}$ patients who underwent resection in the Fourth Affiliated Hospital of Harbin Medical University (Harbin, China). No patient had undergone chemotherapy, radiotherapy or adjuvant treatment prior to surgery. The clinicopathological characteristics of these patients are presented in Table 1. All subjects gave written informed consent, and this study was approved by the Ethics Committee of the Fourth Affiliated Hospital of

Table I Correlation between ZEBI-ASI expression and clinicopathologic features in 106 CC patients

\begin{tabular}{|c|c|c|c|c|}
\hline \multirow[t]{2}{*}{ Features } & \multirow{2}{*}{$\begin{array}{l}\text { Total } \\
\text { number } \\
(n=106)\end{array}$} & \multicolumn{2}{|c|}{$\begin{array}{l}\text { ZEB I-ASI } \\
\text { expression }\end{array}$} & \multirow[t]{2}{*}{$P$-value } \\
\hline & & $\begin{array}{l}\text { Low } \\
(n=47)\end{array}$ & $\begin{array}{l}\text { High } \\
(n=59)\end{array}$ & \\
\hline Age (years) & & & & 0.395 \\
\hline$\leq 50$ & 47 & 23 & 24 & \\
\hline$>50$ & 59 & 24 & 35 & \\
\hline Tumor size $(\mathrm{cm})$ & & & & 0.074 \\
\hline$\leq 4$ & 62 & 32 & 30 & \\
\hline$>4$ & 44 & 15 & 29 & \\
\hline Histological type & & & & 0.854 \\
\hline Squamous cell carcinoma & 78 & 35 & 43 & \\
\hline Adenocarcinoma & 28 & 12 & 16 & \\
\hline FIGO stage & & & & 0.040 \\
\hline lb-Ila & 51 & 30 & 21 & \\
\hline Ilb-IIla & 55 & 17 & 38 & \\
\hline Histological differentiation & & & & 0.071 \\
\hline Well + moderate & 69 & 35 & 34 & \\
\hline Poor & 37 & 12 & 25 & \\
\hline Lymph node metastasis & & & & 0.002 \\
\hline Yes & 41 & 26 & 15 & \\
\hline No & 65 & 21 & 44 & \\
\hline
\end{tabular}

Abbreviations: CC, cervical cancer; ZEBI-ASI, zinc finger E-box binding homeobox I-antisense I.
Harbin Medical University. All the tissue specimens were immediately frozen in liquid nitrogen and stored at $-80^{\circ} \mathrm{C}$ until further analysis.

\section{Cell culture and transfection}

A normal cervical cell line Crl-2614 and three human CC cell lines (HeLa, C33A and $\mathrm{SiHa}$ ) were purchased from American Type Culture Collection (Manassas, VA, USA). All cells were cultured in Dulbecco's Modified Eagle's Medium (DMEM; Thermo Fisher Scientific, Waltham, MA, USA) supplemented with $10 \%$ fetal bovine serum (FBS; Thermo Fisher Scientific) and 1\% penicillin/streptomycin in a humidified incubator with $5 \% \mathrm{CO}_{2}$ at $37^{\circ} \mathrm{C}$. Exponentially growing cells were used for further experiments.

For ZEB1-AS1 overexpression, a full-length human ZEB1-AS1 cDNA was amplified by PCR and inserted into the mammalian expression vector pcDNA3.1(+) (Thermo Fisher Scientific). The empty vector was used as the control. Cells were cultured to $80 \%$ confluence and transfected with the aforementioned vectors using Lipofectamine 2000 (Thermo Fisher Scientific). For ZEB1-AS1 or ZEB1 knockdown, cells were transfected with ZEB1-AS1-specific small interfering RNA (si-ZEB1-AS1), ZEB1-specific siRNA (si-ZEB1) or negative siRNA control (si-NC) (GenePharma, Shanghai, China). Then, 48 hours after transfection, the transfection efficiency was confirmed by RT-qPCR analysis.

\section{RNA extraction and RT-qPCR analysis}

The total RNA from tissue samples or cell lines was extracted using Trizol Reagent (Thermo Fisher Scientific). cDNA was synthesized using the PrimeScript RT reagent Kit (TaKaRa, Dalian, China), and qPCR analysis was then performed using the SYBR Premix Ex Taq ${ }^{\mathrm{TM}}$ II kit (TaKaRa) on an ABI PRISM 7500 fast Sequence Detection System (Thermo Fisher Scientific). Relative gene expression was calculated using $2^{-\Delta \Delta \mathrm{Ct}}$ method. ${ }^{8} \mathrm{GAPDH}$ was used as the internal control.

\section{MTT assay}

Cell proliferation was investigated by 3-(4,5-Dimethylthiazol2-yl)-2,5-diphenyltetrazolium bromide (MTT) assay. In brief, after transfection, cells were seeded into 96-well plates at a cell density of $5 \times 10^{3}$ cells per well and stained at indicated time points with $20 \mu \mathrm{L}$ MTT dye $(5 \mathrm{mg} / \mathrm{mL}$; Sigma-Aldrich Co., St Louis, MO, USA) at $37^{\circ} \mathrm{C}$ for additional 4 hours. Then, the medium was removed and DMSO (Sigma-Aldrich Co.) at $150 \mu \mathrm{L} /$ well was added to dissolve the formazan crystals. The absorbance was determined at $490 \mathrm{~nm}$ using a microplate reader (Molecular Devices LLC, Sunnyvale, CA, USA). 


\section{Cell apoptosis analysis}

The apoptosis rate of cells was detected using AnnexinV/ propidium iodide detection kit (Keygene, Nanjing, China). Briefly, after transfection, cells were harvested and then stained with $5 \mu \mathrm{L}$ Annexin V-FITC and $5 \mu \mathrm{L}$ PI for 15 minutes at $4^{\circ} \mathrm{C}$. Then, the stained cells were analyzed by flow cytometer (BD Biosciences, San Jose, CA, USA).

\section{Transwell assay}

After transfection, a total of $2 \times 10^{5}$ cells in $100 \mu \mathrm{L}$ of serum-free medium were seeded into the upper chambers $(8-\mu \mathrm{m}$ pore size; EMD Millipore, Billerica, MA, USA) coated with or without Matrigel (EMD Millipore). The lower chambers were filled with $500 \mu \mathrm{L}$ of medium containing $10 \%$ FBS. After 48 hours of incubation, the cells remaining on the upper membrane surface were removed, and the cells on the lower membrane surface were fixed with methanol, stained with crystal violet solution and photographed under microscope.

\section{Western blot analysis}

Cells were collected and lysed using RIPA protein extraction reagent (Beyotime, Shanghai, China). Equal amount of protein was separated using SDS-PAGE and transferred to nitrocellulose membranes (GE Healthcare Bio-Sciences Corp., Piscataway, NJ, USA). Following blocking in 5\% fat-free milk for 1 hour, the membranes were incubated at $4^{\circ} \mathrm{C}$ overnight with primary antibodies against E-cadherin (1:2,000; Abcam, Cambridge, MA, USA), N-cadherin (1:2,000; Abcam), Vimentin (1:2,000; Abcam), ZEB1 (1:2,000; Abcam) or GAPDH (1:2,000; Abcam). Then, the membranes were incubated with appropriate secondary antibodies (1:10,000; Santa Cruz Biotechnology Inc., Dallas, TX, USA) at room temperature for 1 hour. Protein bands were visualized using enhanced chemiluminescence reagent (Beyotime). The pixel intensity of each band was quantified using ImageQuant 5.2 software (GE Healthcare Bio-Sciences Corp.). GAPDH was set as the internal control.

\section{In vivo xenografts}

A total of 12 male BALB/c athymic nude mice (6-week old, 18-20 g), purchased from Shanghai SLAC Laboratory Animal, Co., Ltd. (Shanghai, China), were allocated into two groups. CC xenografts in the nude mice were established by subcutaneous injection of $200 \mu \mathrm{L}$ PBS containing $1 \times 10^{6}$ cells of HeLa stably transfected with sh-NC or sh-ZEB1-AS1. Tumor volumes were measured by vernier caliper every 3 days using the following formula: volume $=1 / 2 \times$ length $\times$ width $^{2}$. The mice were killed after 28 days postinjection, and the tumors from the mice were weighed. This in vivo study was approved by the Ethics Committee of the Fourth Affiliated Hospital of Harbin Medical University, and all procedures were performed in accordance with their approved protocols.

\section{Statistical analysis}

All statistical analyses were performed using SPSS 17.0 (SPSS Inc., Chicago, IL, USA) and GraphPad Prism 6.0 (GraphPad Software, Inc., La Jolla, CA, USA). The data were analyzed using independent two-tailed Student's $t$-test. The chi-squared test was used to determine the relationship between ZEB1-AS1 expression and the clinicopathological characteristics of CC patients. We used Kaplan-Meier curve and log-rank test to evaluate the effect of ZEB1-AS1 on survival of CC patients. Correlation between ZEB1-AS1 and ZEB1 mRNA expression in CC tissues was determined by Pearson's correlation analysis. All tests were two-sided, and $P<0.05$ was defined as statistically significant.

\section{Results}

\section{ZEBI-ASI is upregulated in CC tissues}

First, the expression levels of lncRNA ZEB1-AS1 were explored in a cohort of 106 pairs of CC tissues and corresponding adjacent normal tissues. Significantly higher ZEB1AS1 expression was found in CC tissues than in adjacent normal tissues (Figure 1A). We next examined whether ZEB1-AS1 expression was associated with clinical characteristics of CC patients. A total of $106 \mathrm{CC}$ patients were allocated into a low expression group $(<$ mean, $n=47)$ and a high expression group ( $\geq$ mean, $n=59$ ) based on the cutoff value of ZEB1-AS1 expression, and the results showed that higher expression of ZEB1-AS1 was significantly associated with FIGO stage $(P=0.040)$ and lymph node metastasis $(P=0.002)$ (Table 1). Moreover, Kaplan-Meier curve and log-rank results indicated that $\mathrm{CC}$ patients with higher ZEB1-AS1 expression had a poor overall survival $(P=0.035$; Figure 1B). In addition, as shown in Figure 1C, ZEB1-AS1 expression was significantly increased in CC cell lines (HeLa, $\mathrm{C} 33 \mathrm{~A}$ and $\mathrm{SiHa}$ ) compared with that in the normal cervical cell line Crl-2614.

\section{ZEBI-ASI promotes CC cell proliferation in vitro}

To further analyze the effects of ZEB1-AS1 on the malignant phenotypes of $\mathrm{CC}$ cells, gain and loss function assays were then performed. RT-qPCR analysis showed that transfection of si-ZEB1-AS1 significantly suppressed ZEB1-AS1 expression in HeLa cells, and transfection of pcDNA3.1-ZEB1-AS1 obviously increased ZEB1-AS1 expression in C33A cells 
A

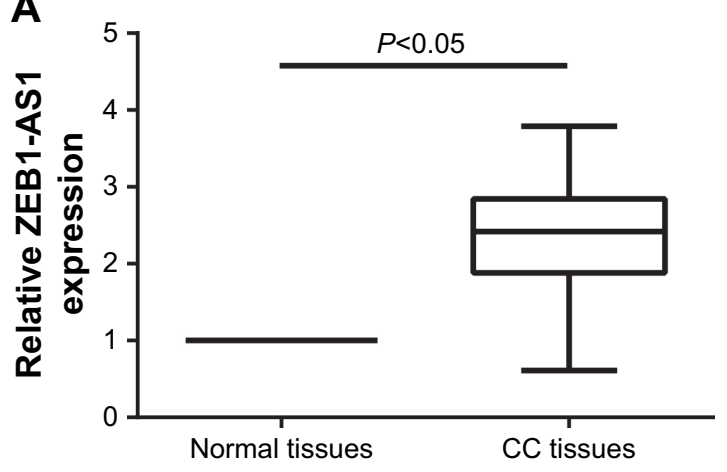

B

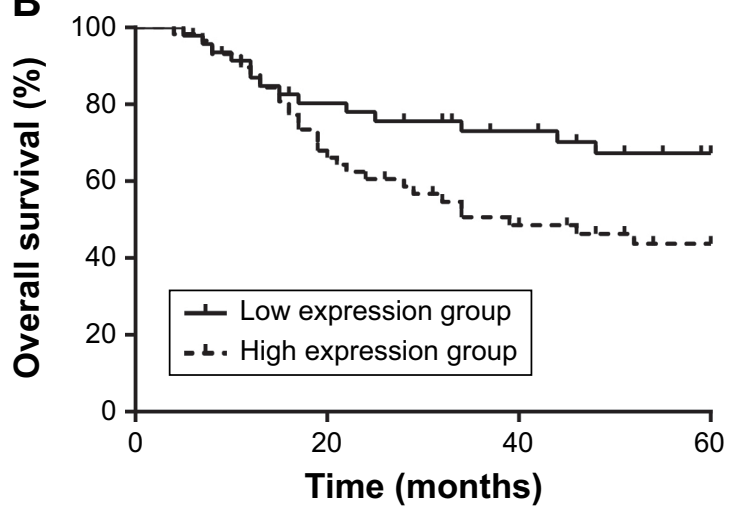

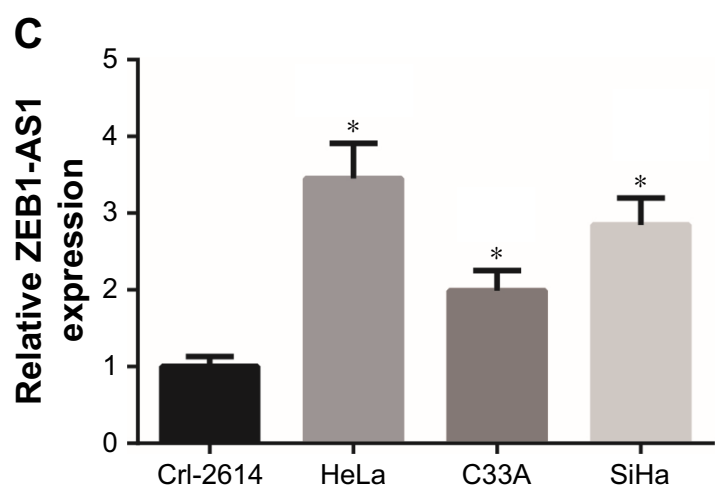

Figure I ZEBI-ASI is upregulated in CC tissues.

Notes: (A) RT-qPCR analysis of ZEBI-ASI expression levels in 106 pairs of CC and corresponding noncancerous tissues. (B) Kaplan-Meier curve showing overall survival in CC patients stratified by ZEBI-ASI expression. (C) RT-qPCR analysis of ZEBI-ASI expression levels in three human CC cell lines. The data represent the mean \pm SD. $* P<0.05$ vs $\mathrm{Crl}-26 \mathrm{I} 4 \mathrm{cells}$.

Abbreviations: CC, cervical cancer; ZEBI-ASI, zinc finger E-box binding homeobox I-antisense I.

(Figure 2A). The results of MTT assay demonstrated that the proliferation of HeLa cells was significantly inhibited upon ZEB1-AS1 knockdown, whereas ZEB1-AS1 overexpression enhanced the proliferation of C33A cells (Figure 2B). Moreover, we found that transfection of si-ZEB1-AS1 remarkably increased the number of apoptotic HeLa cells (Figure 2C).

\section{Knockdown of ZEBI-ASI inhibits CC xenograft growth in vivo}

To further determine the effects of ZEB1-AS1 on CC tumorigenesis in vivo, we then analyzed the effects of ZEB1-AS1 on the CC xenograft growth in nude mice. As shown in Figure 3A, tumors in the sh-ZEB1-AS1 group grew much more slowly. The average tumor weight of the sh-ZEB1-AS1 group was also reduced compared with that of the sh-NC group (Figure 3B). Moreover, as expected, we found that the expression of ZEB1AS1 was remarkably reduced in the xenograft tissues derived from sh-ZEB1-AS1-transfected cells (Figure 3C).

\section{ZEBI-ASI promotes CC cell invasion and EMT in vitro}

Transwell assay was then employed to gain an insight into the role of ZEB1-AS1 on the migration and invasion of $\mathrm{CC}$ cells. As presented in Figure 4A, the number of migrated and invaded HeLa cells was significantly reduced after transfection of si-ZEB1-AS1, and overexpression of ZEB1-AS1 significantly enhanced the migratory and invasive capacities of C33A cells. Moreover, we also evaluated the expression levels of EMT-related proteins. The results of Western blot analysis showed that ZEB1-AS1 knockdown induced the expression of E-cadherin, but reduced the expression of $\mathrm{N}$-cadherin and Vimentin in HeLa cells. In contrast, the expression of E-cadherin was reduced, whereas the expression levels of $\mathrm{N}$-cadherin and Vimentin were increased in C33A cells with ZEB1-AS1 overexpression (Figure 4B).

\section{ZEBI-ASI increases ZEBI expression in CC cells}

ZEB1 is an important EMT promoter in cancer cells. ${ }^{9}$ We then analyzed whether ZEB1-AS1 regulates ZEB1 expression in CC cells. As demonstrated in Figure 5A and B, knockdown of ZEB1-AS1 markedly reduced the mRNA and protein levels of ZEB1 in HeLa cells, whereas overexpression of ZEB1-AS1 markedly increased the mRNA and protein levels of ZEB1 in C33A cells. To further validate the relationship between ZEB1-AS1 and ZEB1 in CC, we analyzed 

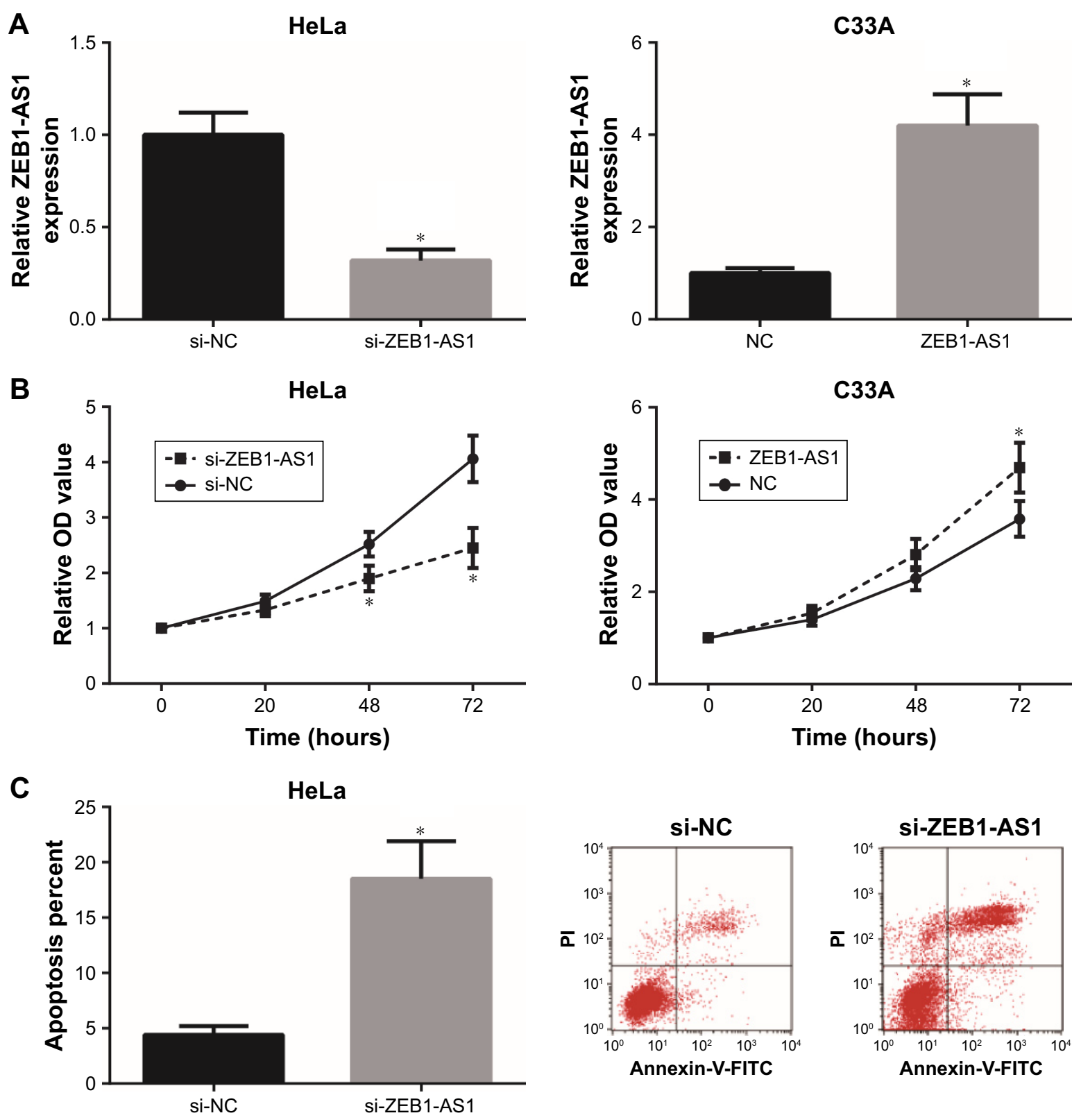

Figure 2 ZEBI-ASI promotes CC cell proliferation in vitro.

Notes: (A) RT-qPCR analysis of ZEBI-ASI expression levels in HeLa and C33A cells after transfection. (B) The proliferation of HeLa and C33A cells after transfection was determined by MTT assay. (C) The apoptosis of HeLa and C33A cells after transfection was detected by flow cytometric analysis. The data represent the mean \pm SD. $* P<0.05$ vs si-NC-transfected HeLa cells or empty vector-transfected C33A cells.

Abbreviations: CC, cervical cancer; si-NC, negative siRNA control; si-ZEBI-ASI, ZEBI-ASI-specific small interfering RNA; ZEBI-ASI, zinc finger E-box binding homeobox I-antisense I.

the correlation between ZEB1-AS1 and ZEB1 expression in $\mathrm{CC}$ tissues. We found that the expression of ZEB1 mRNA in $\mathrm{CC}$ tissues was also significantly increased compared to corresponding normal tissues (Figure 5C), which was positively correlated with the expression of ZEB1-AS1 (Figure 5D).

\section{Knockdown of ZEBI abrogates the oncogenic role of ZEBI-ASI in CC cells}

Subsequently, we analyzed whether ZEB1 is implicated in the oncogenic role of ZEB1-AS1 in CC cells. As demonstrated in Figure 6, knockdown of ZEB1 obviously attenuated the migratory and invasive abilities of C33A cells with ZEB1AS1 overexpression.

\section{Discussion}

The mechanisms underlying $\mathrm{CC}$ are complicated and involve dysregulation of a number of oncogenes and tumor suppressors. IncRNAs were initially regarded as largely useless transcription (transcriptional "noise"), ${ }^{10}$ but currently, more and more lncRNAs were emerging as pivotal regulators 
A
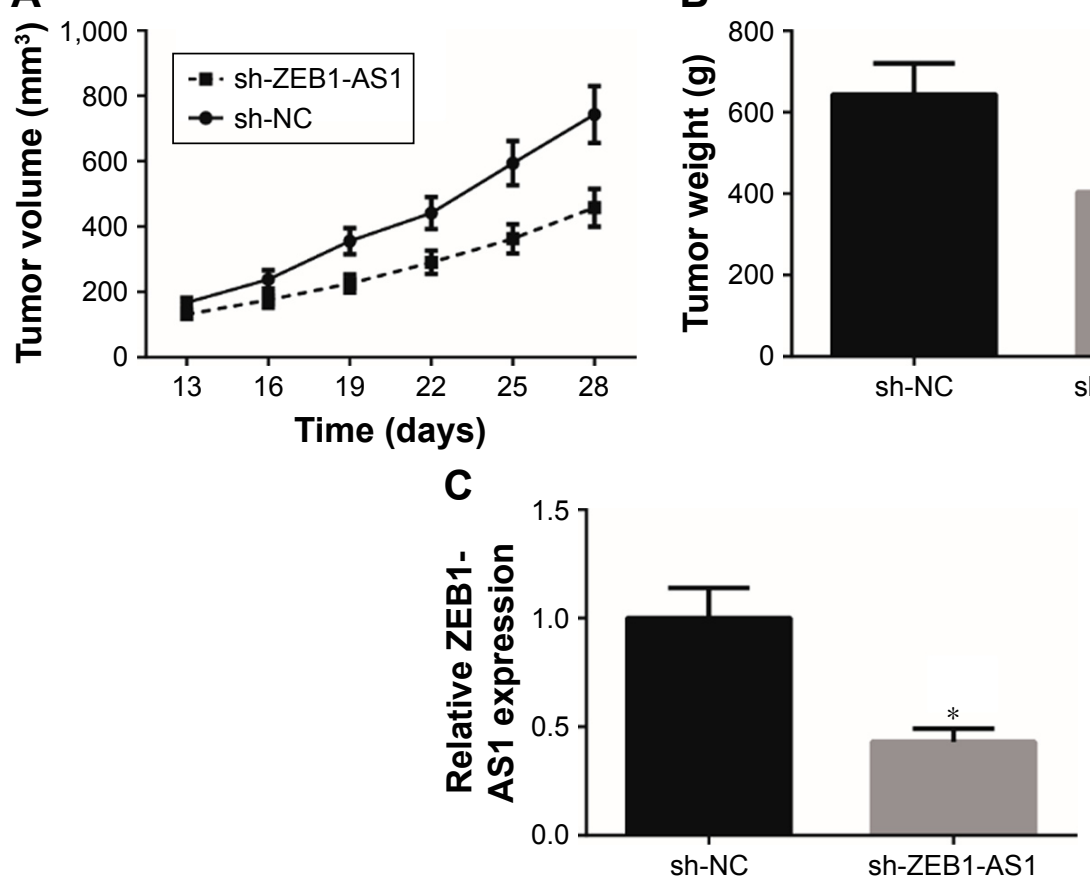

sh-NC

sh-ZEB1-AS1
B

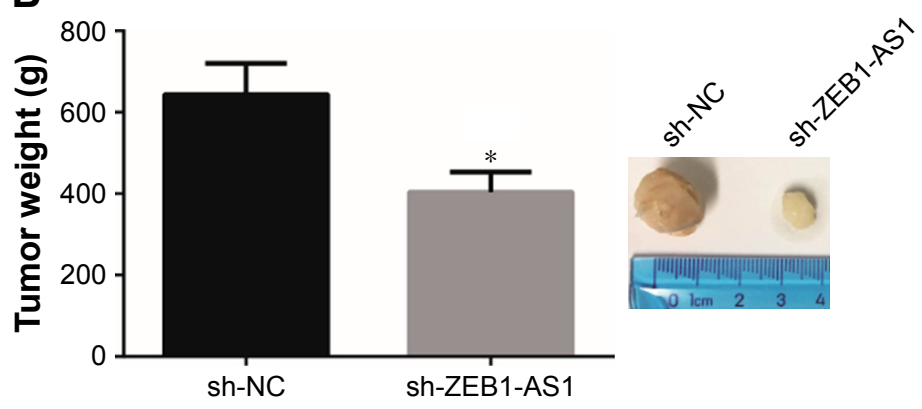

Figure 3 Knockdown of ZEBI-ASI inhibits CC xenograft growth in vivo.

Notes: (A) The tumor volume was measured every 3 days after cell implantation. (B) The average weight of the tumors. (C) RT-qPCR analysis of ZEBI-ASI expression levels in CC xenograft tissues. The data represent the mean \pm SD. $* P<0.05$ vs sh-NC group.

Abbreviations: CC, cervical cancer; ZEBI-ASI, zinc finger E-box binding homeobox I-antisense I.

in the oncogenesis and progression of CC. ${ }^{11}$ For example, knockdown of lncRNA PCAT-1 attenuated the proliferation and invasion of CC cells. ${ }^{12}$ Moreover, lncRNA FEZF1-AS1 is upregulated and associated with poor prognosis in $\mathrm{CC}$ patients. ${ }^{13}$ Accordingly, systematically exploring the functions of CC-related lncRNAs may help identify novel promising therapeutic strategies for the treatment of CC.

ZEB1-AS1 is identified as an oncogenic regulator in diverse malignancies. ${ }^{14}$ The purpose of the present study was to analyze the biological significance of ZEB1-AS1 for CC. The results demonstrated that ZEB1-AS1 was overexpressed in $\mathrm{CC}$ and might serve as a valuable prognostic indicator for $\mathrm{CC}$ patients. Further experimental data indicated that knockdown of ZEB1-AS1 inhibited and overexpression of it promoted the proliferation, migration and invasion of CC cells in vitro. The in vivo study also showed that knockdown of ZEB1-AS1 could reduce the volume and weight of $\mathrm{CC}$ xenografts in nude mice. All these findings confirmed the oncogenic role of ZEB1-AS1 in the carcinogenesis of CC.

The process of cancer metastasis consists of several steps. EMT facilitates cancerous epithelial cells to enter into a mesenchymal-like state, and therefore, EMT is considered as an important step for the invasion and metastasis of malignant tumors. ${ }^{15,16}$ Recent articles outline the involvement of
ZEB1-AS1 in the induction and regulation of EMT. In glioma and gastric cancer, ZEB1-AS1 serves as a regulator of cell migration and invasion via activating EMT. ${ }^{5,7}$ EMT also plays a critical role in CC progression and metastasis. ${ }^{17} \mathrm{We}$ showed here that ZEB1-AS1 knockdown inhibited EMT by inducing upregulation of E-cadherin and downregulation of $\mathrm{N}$-cadherin and Vimentin in CC cells.

ZEB1 is a transcription factor that promotes tumor invasion and metastasis by inducing EMT. ${ }^{9}$ Aberrant expression of ZEB1 has been reported in many human cancers. In hepatocellular carcinoma, ZEB1-AS1 positively regulates the expression of ZEB1, and ZEB1 inhibition partially abrogates ZEB1-AS1-induced EMT. ${ }^{18}$ The involvement of ZEB1 in CC development has been frequently studied, ${ }^{19,20}$ and our study also showed that ZEB1 mRNA expression was positively correlated with ZEB1-AS1 expression in human $\mathrm{CC}$ tissues, and knockdown of ZEB1 blocked the oncogenic role of ZEB1-AS1 in CC cells.

Collectively, the present study for the first time demonstrated that ZEB1-AS1 might promote CC tumorigenesis and progression, partly through regulating ZEB1. Our findings might provide wider insight into the molecular mechanisms underlying $\mathrm{CC}$ aggression, and ZEB1-AS1 could be considered as a potential therapeutic target for $\mathrm{CC}$ in the near future. 
A

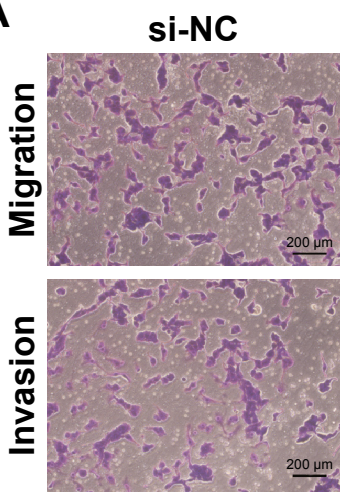

NC

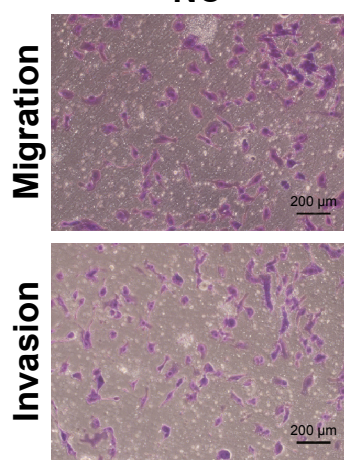

B
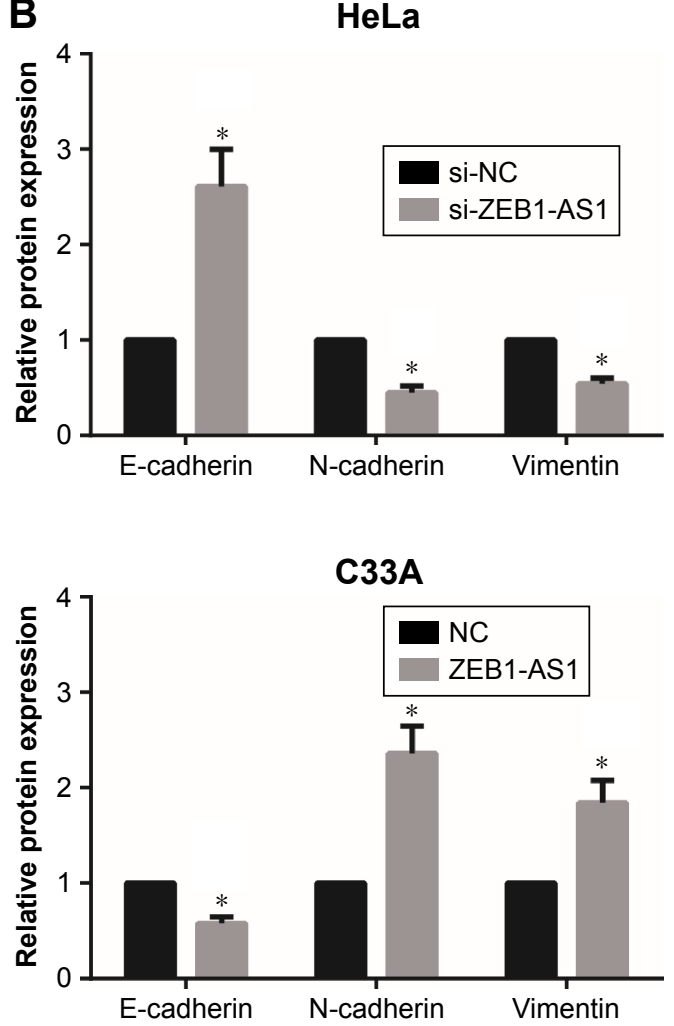

HeLa

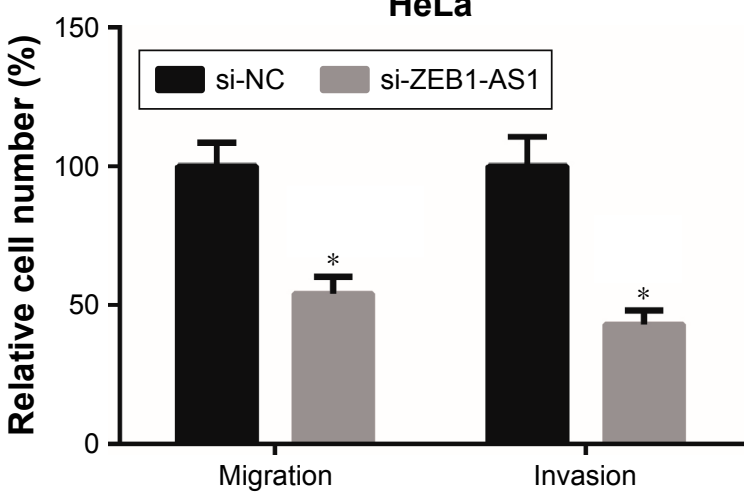

C33A
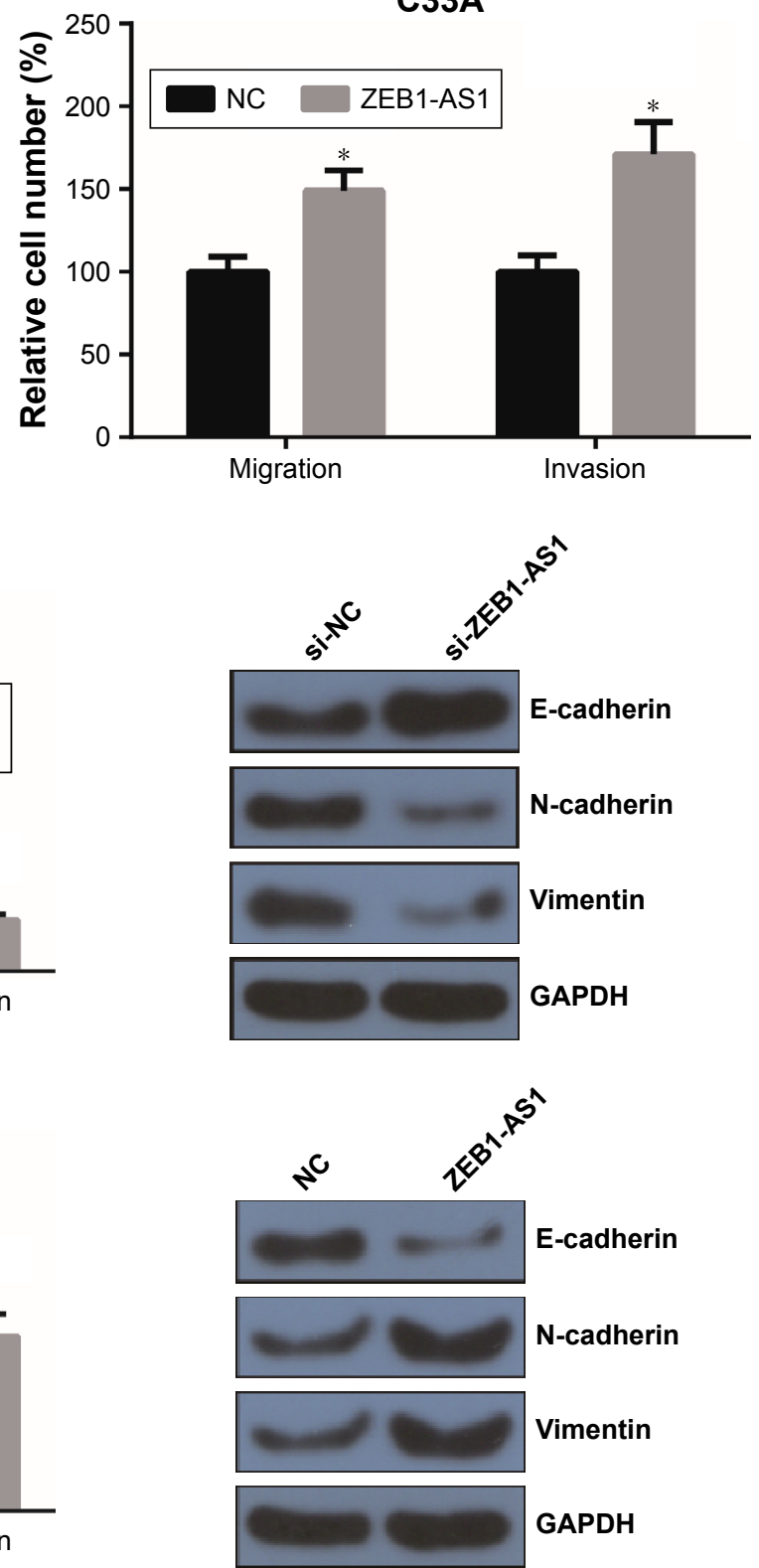

Figure 4 ZEBI-ASI promotes CC cell invasion and EMT in vitro.

Notes: (A) The migration and invasion of HeLa and C33A cells after transfection were determined by transwell assay. (B) The protein expression levels of E-cadherin, $\mathrm{N}$-cadherin and Vimentin in HeLa and C33A cells after transfection were detected by Western blot analysis. The data represent the mean \pm SD. $* P<0.05$ vs si-NC-transfected HeLa cells or empty vector-transfected C33A cells.

Abbreviations: CC, cervical cancer; EMT, epithelial-mesenchymal transition; si-NC, negative siRNA control; si-ZEBI-ASI, ZEBI-ASI-specific small interfering RNA; ZEBIASI, zinc finger E-box binding homeobox I-antisense I. 
A

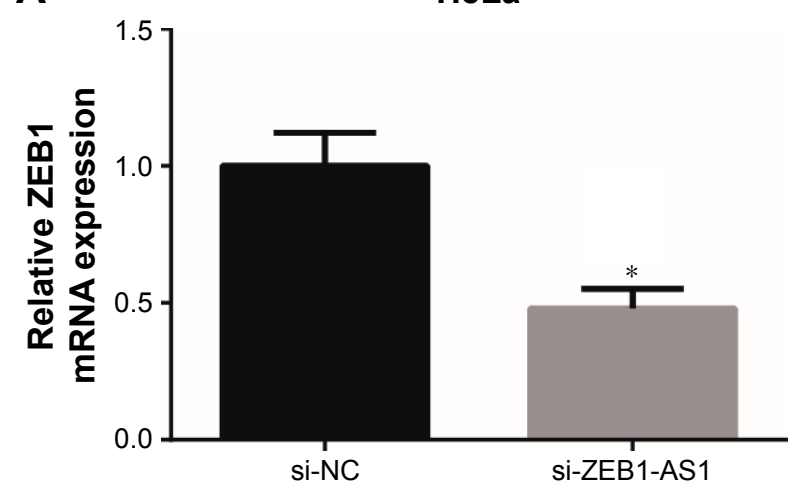

B

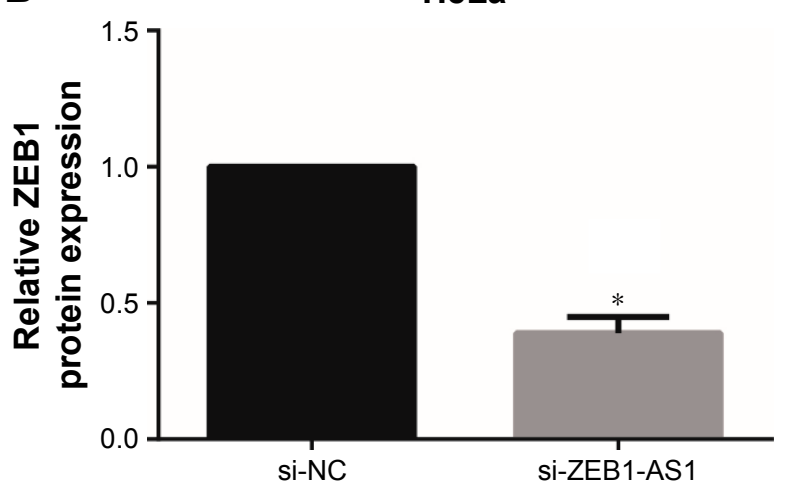

C33A

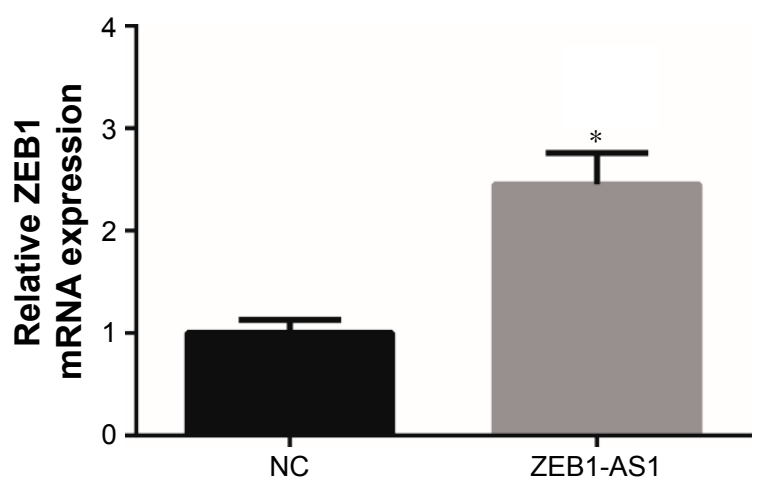

C33A

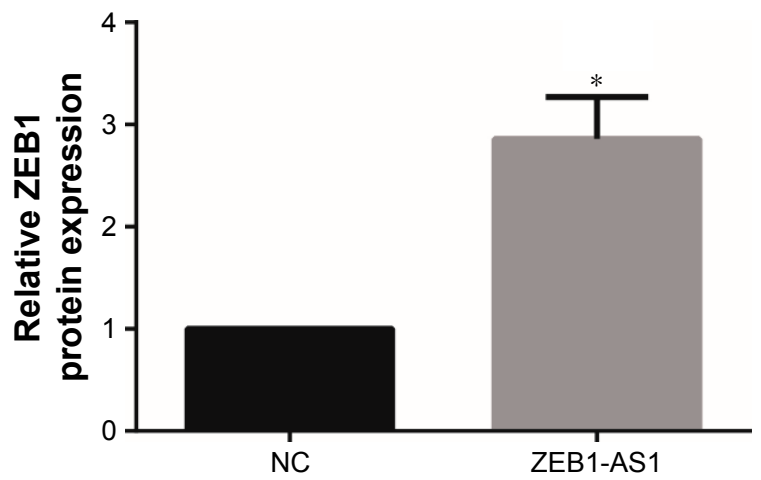

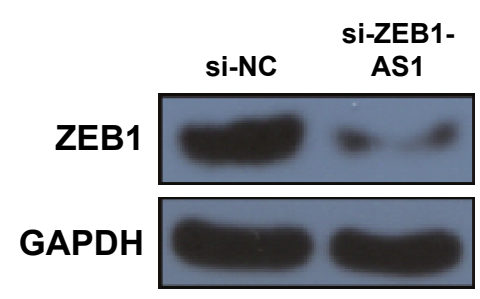

HeLa

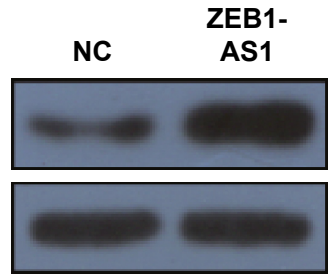

C33A
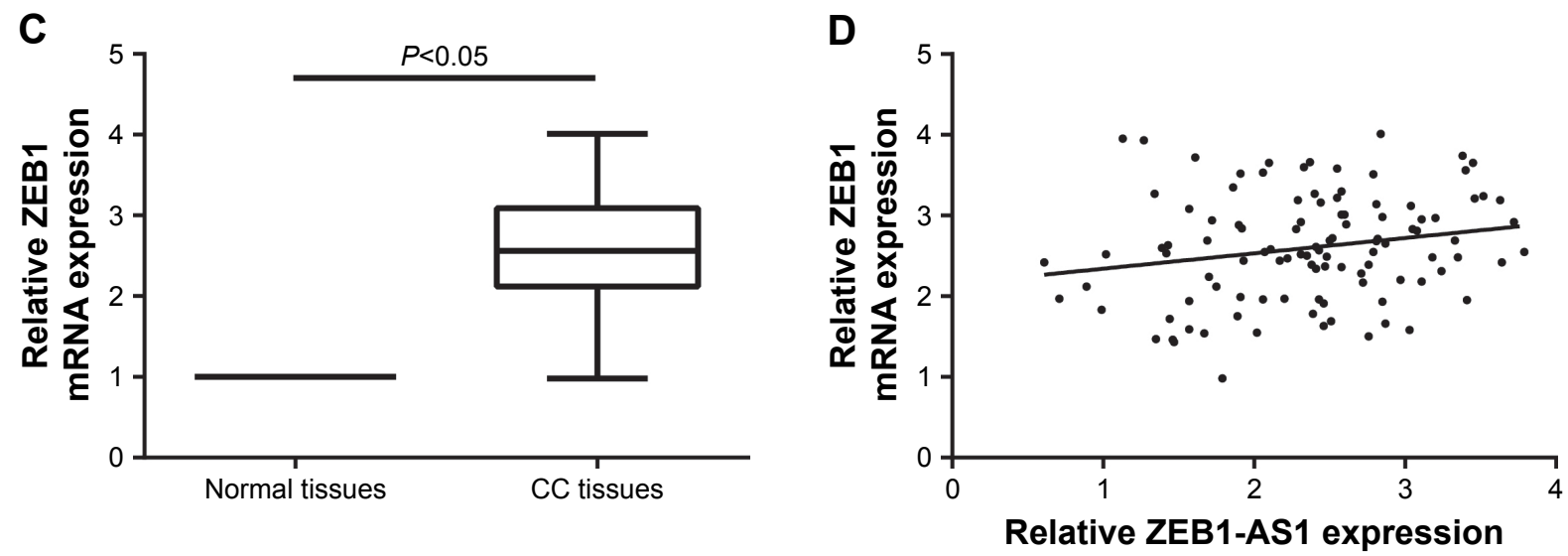

Figure 5 ZEBI-ASI increases ZEBI expression in CC cells.

Notes: (A) RT-qPCR analysis of ZEBI mRNA expression levels in HeLa and C33A cells after transfection. (B) Western blot analysis of ZEBI protein expression levels in HeLa and C33A cells after transfection. The data represent the mean \pm SD. $* P<0.05$ vs si-NC-transfected HeLa cells or empty vector-transfected C33A cells. (C) RT-qPCR analysis of ZEBI mRNA expression levels in CC and corresponding noncancerous tissues. (D) The correlation between ZEBI-ASI and ZEBI mRNA expression in CC tissues was analyzed by Pearson's correlation analysis.

Abbreviations: CC, cervical cancer; si-NC, negative siRNA control; si-ZEBI-ASI, ZEBI-ASI-specific small interfering RNA; ZEBI-ASI, zinc finger E-box binding homeobox I-antisense I. 


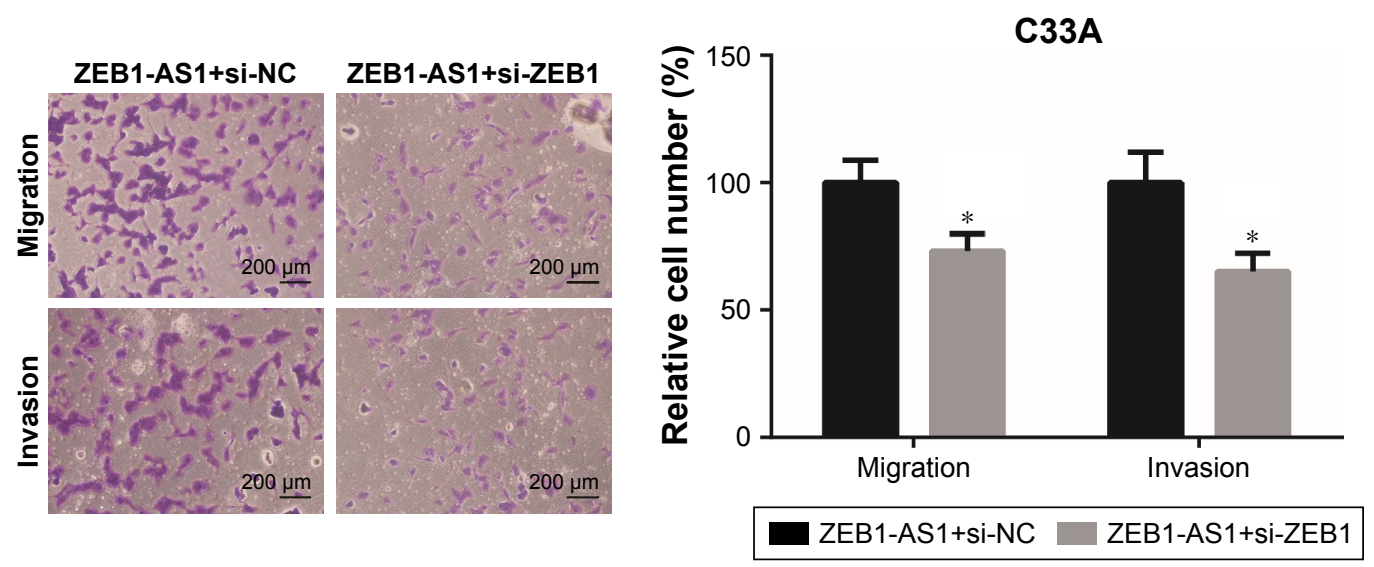

Figure 6 Knockdown of ZEBI abrogates the oncogenic role of ZEBI-ASI in CC cells.

Notes: The migration and invasion of C33A cells after transfection were determined by transwell assay. The data represent the mean \pm SD. $* P<0.05$ vs $Z E B I-A S I+$ si-NCcotransfected $C 33 \mathrm{~A}$ cells.

Abbreviations: CC, cervical cancer; si-NC, negative siRNA control; si-ZEBI, ZEBI-specific siRNA; ZEBI-ASI, zinc finger E-box binding homeobox I-antisense I.

\section{Acknowledgment}

We thank the financial support from Heilongjiang Youth Science Foundation (QC2018100).

\section{Disclosure}

The authors report no conflicts of interest in this work.

\section{References}

1. Jemal A, Bray F, Center MM, Ferlay J, Ward E, Forman D. Global cancer statistics. CA Cancer J Clin. 2011;61(2):69-90.

2. Diaz-Padilla I, Monk BJ, Mackay HJ, Oaknin A. Treatment of metastatic cervical cancer: future directions involving targeted agents. Crit Rev Oncol Hematol. 2013;85(3):303-314.

3. Wapinski O, Chang HY. Long noncoding RNAs and human disease. Trends Cell Biol. 2011;21(6):354-361.

4. Huarte M. The emerging role of lncRNAs in cancer. Nat Med. 2015; 21(11):1253-1261.

5. Lv QL, Hu L, Chen SH, et al. A long noncoding RNA ZEB1-AS1 promotes tumorigenesis and predicts poor prognosis in glioma. Int $J$ Mol Sci. 2016;17(9):E1431.

6. Fu J, Cui Y. Long noncoding RNA ZEB1-AS1 expression predicts progression and poor prognosis of colorectal cancer. Int J Biol Markers. 2017;32(4):e428-e433.

7. Li Y, Wen X, Wang L, et al. LncRNA ZEB1-AS1 predicts unfavorable prognosis in gastric cancer. Surg Oncol. 2017;26(4):527-534.

8. Livak KJ, Schmittgen TD. Analysis of relative gene expression data using real-time quantitative PCR and the 2(-Delta Delta C(T)) Method. Methods. 2001;25(4):402-408.

9. Zhang P, Sun Y, Ma L. ZEB1: at the crossroads of epithelial-mesenchymal transition, metastasis and therapy resistance. Cell Cycle. 2015;14(4): $481-487$.

OncoTargets and Therapy

\section{Publish your work in this journal}

OncoTargets and Therapy is an international, peer-reviewed, open access journal focusing on the pathological basis of all cancers, potential targets for therapy and treatment protocols employed to improve the management of cancer patients. The journal also focuses on the impact of management programs and new therapeutic agents and protocols on
10. Wilusz JE, Sunwoo H, Spector DL. Long noncoding RNAs: functional surprises from the RNA world. Genes Dev. 2009;23(13):1494-1504.

11. Peng L, Yuan X, Jiang B, Tang Z, Li GC. LncRNAs: key players and novel insights into cervical cancer. Tumour Biol. 2016;37(3): 2779-2788.

12. Ma TT, Zhou LQ, Xia JH, Shen Y, Yan Y, Zhu RH. LncRNA PCAT-1 regulates the proliferation, metastasis and invasion of cervical cancer cells. Eur Rev Med Pharmacol Sci. 2018;22(7):1907-1913.

13. Zhang HH, Li AH. Long non-coding RNA FEZF1-AS1 is up-regulated and associated with poor prognosis in patients with cervical cancer. Eur Rev Med Pharmacol Sci. 2018;22(11):3357-3362.

14. Li J, Li Z, Leng K, et al. ZEB1-AS1: a crucial cancer-related long noncoding RNA. Cell Prolif. 2018;51(1):12423.

15. Thiery JP. Epithelial-mesenchymal transitions in tumour progression. Nat Rev Cancer. 2002;2(6):442-454.

16. Thiery JP, Acloque H, Huang RY, Nieto MA. Epithelial-mesenchymal transitions in development and disease. Cell. 2009;139(5):871-890

17. Qureshi R, Arora H, Rizvi MA. EMT in cervical cancer: its role in tumour progression and response to therapy. Cancer Lett. 2015;356(2 Pt B): $321-331$.

18. Li T, Xie J, Shen C, et al. Upregulation of long noncoding RNA ZEB1-AS1 promotes tumor metastasis and predicts poor prognosis in hepatocellular carcinoma. Oncogene. 2016;35(12):1575-1584.

19. Ran J, Lin DL, Wu RF, et al. ZEB1 promotes epithelial-mesenchymal transition in cervical cancer metastasis. Fertil Steril. 2015;103(6): 1606-1614.e1-2.

20. Ma Y, Zheng X, Zhou J, Zhang Y, Chen K. ZEB1 promotes the progression and metastasis of cervical squamous cell carcinoma via the promotion of epithelial-mesenchymal transition. Int J Clin Exp Pathol. 2015;8(9):11258-11267.

patient perspectives such as quality of life, adherence and satisfaction The manuscript management system is completely online and includes a very quick and fair peer-review system, which is all easy to use. Visit http://www.dovepress.com/testimonials.php to read real quotes from published authors. 